\title{
GLI STEREOTIPI DEI RUSSI SULL'ITALIA E GLI ITALIANI
}

\section{Analisi di un corpus di barzellette russe contemporanee}

\section{Laura Salmon}

doi: http://dx.doi.org/10.7359/834-2017-salm

«Se hai da ammazzare qualcuno, scrivimi, arrivo!»

(Črezvyčajnye priključenija ital'jancev $v$ Rossii)

\section{Premesse teoriche}

\subsection{Comicità vs umorismo: le macro-categorie della derisione verbale}

Il riso e la derisione sono considerate risposte adattive della specie umana e, per di più, una delle facoltà più sofisticate:

There are several reasons to suppose humor and laughter could be evolutionarily adaptive $[. .$.$] . Given that even a simple joke can utilize language skills,$ theory-of-mind, symbolism, abstract thinking, and social perception, humor may arguably be humankind's most complex cognitive attribute. (Polimeni Reiss 2006, 348)

Non è possibile sapere se il riso e la derisione si siano evoluti con e grazie al linguaggio, anche se, probabilmente, le forme più sofisticate di derisione sono connesse all'uso delle tecniche verbali:

One does not need words to convey humor; however, conversation greatly enhances the opportunity for humorous expression. Consequently, humor usually utilizes a string of complex symbols (words). (ivi, 359)

In numerose lingue, il fenomeno della derisione è genericamente definito col termine polisemico e contraddittorio di bumour, il quale comprende in realtà due macro-categorie di derisione che, dal punto di vista funzionale e strutturale, sono in opposizione: la comicità, da una parte, e l'umorismo 
dall'altra. Come già nel 1898 aveva anticipato Theodor Lipps (2012, 227), la comicità (Komik) è in sostanza il contrario dell'umorismo (Humor) ${ }^{1}$. Nel noto saggio L'umorismo del 1908, Luigi Pirandello dimostrava poi che la derisione comica è basata su stereotipi retorici ed è quindi fondamentalmente conservativa, mentre l'umorismo ha la funzione opposta di smascherare e smantellare gli stereotipi, innescando un empatico «sentimento del contrario» (Pirandello 1995, 173 ss.), portando cioè chi deride a riflettersi in chi è deriso ${ }^{2}$. Se l'umorismo è empatico e paradossale, e nega la logica del senso comune e apre la mente a una riflessione esistenziale, la comicità, al contrario, è priva di condivisione affettiva, segue una logica rigida e predispone al rinforzo degli stereotipi (Salmon 2003; 2004a; 2008, 80-100). Per questo, l'umorismo è raro e raffinato, mentre la comicità è diffusa «in gran copia» (Pirandello 1995, 39) presso tutte le nazioni, sia nelle letterature, sia nella vita quotidiana.

Le barzellette appartengono alla macro-categoria della comicità retorica e, sotto forma di scherzi, parodie e facezie, possono essere fatte risalire ai tempi dell'antica Grecia (cf. Bremmer 2005², 11-16; Polimeni - Reiss $2006,348)^{3}$. Tra le ragioni del successo universale di questi testi vi sono: la semplicità strutturale, la brevità, la quantità, l'oralità, la traducibilità, l'anonimato, il fatto che chiunque racconti barzellette può modificare a piacimento il testo narrato da altri (cf. Davies 2005, 70). Trattandosi, quindi, di una narrativa presenziale e performativa (Driessen $2005^{2}, 224$ ), stabilire uno stemma dei testi e risalire all'autore originario della barzelletta è quasi impossibile, così come è difficile pervenire all'«archetipo» più produttivo che ne ha decretato la diffusione. Pertanto, sul piano del rigore, la modalità più consona allo studio delle barzellette è l'analisi delle immagini stereotipiche che ne costituiscono, come si vedrà, l'ossatura. Infatti, questa tipologia testuale rivela come fondamentale funzione sociale quella di stabilire una sorta di esprit de corps (ivi, 237) e, nella triangolazione derisore-deriso-

1 È sorprendente come questa intuizione così rilevante sia stata completamente trascurata anche dai massimi teorici e studiosi degli ultimi decenni.

2 La scarsa attenzione alla teoria pirandelliana porta la maggior parte degli autori ad attribuire a categorie opposte le stesse funzioni. Rocco de Biasi (2006, 107), ad esempio, afferma che «è arduo rinvenire una disposizione filosofica nell'umorismo», mentre in realtà l'umorismo scettico e orientale (ad esempio Zen) è radicalmente filosofico e antitetico al funzionamento delle barzellette e alla comicità (cf. Salmon 2008, 33 ss., 143-148).

3 Il termine italiano «barzelletta», associato al nome di un componimento poetico diffuso tra XV e XVI secolo (cf. Ramous 1984, 92-94), può essere verosimilmente collegato (nel senso di «facezia») all'espressione «azione da bargello», «cioè 'misfatto, birichinata, imbroglio'» (Cortelazzo - Zolli 1992, 119). 
ascoltatore, riflette la posizione pregiudiziale di contrapposizione e superiorità del derisore rispetto a chi è deriso, sfruttando stereotipi condivisi all'interno del proprio gruppo socio-etnico.

La contrapposizione su cui si basa la derisione è binaria: chi è deriso è visto in modo negativo, mentre chi deride rappresenta il valore positivo opposto (ad esempio, gli italiani sono «furbi» e deridono i tedeschi «ottusi» ecc.). Le barzellette «etniche», in particolare, si basano sulla contrapposizione pregiudiziale tra «gli altri - stranieri» (negativo) e «noi stessi» (positivo), ovvero sugli stereotipi attribuiti ai diversi gruppi etnici, talvolta non uno soltanto ${ }^{4}$. Per usare la terminologia di Teun van Dijk $(2014,20)$, il gruppo che assume il ruolo degli «altri» è un outgroup che si contrappone all'ingroup («noi») con cui si identifica il derisore. Per esempio, i francesi sono l'ingroup che deride $\mathrm{i}$ «belgi» (outgroup), basandosi sullo stereotipo della «stupidità» belga, contrapposta alla «non-stupidità» francese.

Pregiudizi e stereotipi su ingroup e outgroup non sono generalizzazioni statistiche, poiché si fondano su pseudo-conoscenze e corollari trasmessi senza verifica da linguaggio e discorso. Le barzellette promuovono pregiudizi e stereotipi che prescindono dai dati dell'esperienza diretta, rafforzando il principio del «tutti sanno che». Chi narra barzellette, dunque, alimenta gli stereotipi sugli «altri» e sul proprio ingroup culturale, etnico o nazionale, rafforzando la condivisione. Grazie alla reazione di divertimento/riso che innescano le barzellette, allo stereotipo viene associato un senso di piacere che lo rinforza (cf. Suls 1976, 118). Il meccanismo è circolare: più una barzelletta è creativa (più fa ridere), maggiore è il rinforzo del motore pregiudiziale che l'ha originata.

\subsection{Stereotipi, pregiudizi, script}

Le definizioni di «pregiudizio» e «stereotipo» sono complesse e diversificate, ma possono essere ricondotte ad alcune formule sintetiche che ne enfatizzano l'impermeabilità al ruolo dell'esperienza e delle argomentazioni ${ }^{5}$. Un

4 In URSS, oltre al filone sulla stupidità dei čukči (eschimesi del nord-est asiatico sovietico) e quello sulla lentezza degli estoni, era diffuso un ampio filone di barzellette sui georgiani, di cui si derideva la munificenza e la prestanza sessuale. Pur trattandosi di qualità invidiabili anche in un regime socialista, la caricatura indicava che la posizione del derisore era comunque sostanzialmente di distinzione (superiorità) rispetto all'oggetto deriso (focosità e ricchezza, infatti, erano qualità poco in auge in epoca sovietica).

5 Il settore della psicologia sociale che studia l'origine e i meccanismi di azione di pregiudizi e stereotipi nel comportamento di individui e gruppi sociali - detto «Psicologia 
pensiero, infatti, diventa pregiudizio quando resta irreversibile alla luce di nuove conoscenze (cf. Allport $1976^{2}, 14-15$ ), ovvero quando è un giudizio «precedente all'esperienza o in assenza di dati empirici» (Mazzara 1997, 14). La differenza tra «pregiudizio» e «generalizzazione» è fondamentale: per generalizzare si deve disporre di «dati inconfutabili concernenti l'esistenza (o l'alta probabilità) di vere differenze di gruppo» (ivi, 268), viceversa, il pregiudizio si avvale solo della premessa dogmatica «tutti sanno che...».

Rispetto ai pregiudizi, gli stereotipi sono più esplicitamente legati al processo di categorizzazione cognitiva e affettiva, ovvero sono credenze valutative relative ad «alcuni gruppi sociali, ad esempio, le donne, gli uomini, gli italiani», sono «immagini molto semplificate e condivise a livello collettivo» (ivi, 11). Come suggerisce l'etimo, il termine «stereotipo» indica rigidità e riflette la tendenza umana a «definire prima di osservare» (Villano 2013², 14; corsivo nell'originale). Accumulandosi, gli stereotipi, trasformano i pregiudizi in categorie valutative che aiutano a giustificare idee irrazionali su un gruppo di persone: la loro funzione è proprio quella «di giustificare (razionalizzare) la nostra condotta in relazione a quella categoria» (Allport 1976², 266).

La predisposizione agli stereotipi è un universale della cognizione umana e non va di per sé considerata una «patologia» (Pickering 2001, 22): non è affatto detto che gli stereotipi siano sempre «falsi», possono anche essere «veri» (qualora li si verifichi con indagini statistiche); né sono necessariamente negativi, poiché servono a categorizzare la realtà in modo economico e in tutte le sue forme. Tuttavia, gli stereotipi etnici sono particolarmente impermeabili ai dati razionali e assecondarli implica allontanarsi da più corrette generalizzazioni. Per esempio, uno dei più diffusi stereotipi russi sugli italiani è che il nostro popolo pratichi molto sesso e (di conseguenza) faccia molti figli (cf. in seguito gli esempi [18] e [19]), mentre i dati statistici informano che da decenni l'Italia è uno dei Paesi con la natalità più bassa al mondo e in continuo calo. Non solo gli stereotipi etnici sono tra i più obsoleti, ma sono spesso grossolanamente approssimativi; all'estero, per esempio, gli «italiani» sono per lo più identificati con i siciliani o i napoletani, e sono considerati «sporchi» (arretrati e incivili), laddove l'igiene è un chiaro simbolo di progresso e civiltà (cf. Davies 2005, 79-82).

dei tratti» - è stato inaugurato negli anni Cinquanta del secolo scorso dal cognitivista americano Gordon W. Allport con The Nature of Prejudice (1954, cf. Allport 1976²). L'altra disciplina che si occupa di stereotipia è quella della Communication research (cf. ad esempio Pickering 2001). 
Gli stereotipi si diffondono in modalità memetica (per replicazione e «contagio») attraverso la comunicazione verbale: gli umani «costruiscono le categorie nel discorso» (Villano 20132 ${ }^{2}$ 107; corsivo nell'originale). Al processo di categorizzazione di un ingroup concorrono le «azioni discorsive» che servono a «difendere la propria immagine di gruppo, colpevolizzare gli altri, sottolineare la diversità culturale e/o la distanza sociale o delegittimare» (ivi, 108). Per dirla con Mazzara $(1997,85)$, il linguaggio non rappresenta semplicemente un veicolo per la diffusione di stereotipi nati altrove, bensì ne è «la sede propria e in definitiva la loro sostanza». Inoltre, come osserva van Dijk $(2014,47)$, parlare significa esprimere modelli mentali e comprendere significa costruire o aggiornare questi stessi modelli. Ciò vale in modo particolare per esprimere a parole le «informazioni fondamentali» con cui i membri di un gruppo categorizzano la propria identità, le proprie norme e, soprattutto, il modo in cui si relazionano con gli altri (ivi, 75).

Le barzellette etniche non solo costituiscono uno dei veicoli più efficaci per la diffusione e il consolidamento degli stereotipi etnici, ma ne sono uno dei «generatori» più potenti e concorrono a strutturare credenze false o tendenziose. Queste credenze, a loro volta, alimentano il pensiero pregiudiziale e privano il singolo individuo della possibilità di sottrarsi alla categorizzazione, secondo un algoritmo rigido:

$$
\text { se }[\mathrm{X}] \text {, allora }[\mathrm{Y}]
$$

ad esempio:

$$
\text { se [genovese, ebreo, scozzese], allora [tirchio] }
$$

Una volta comparsi e radicatisi in una cultura, gli stereotipi si intrecciano con i dati noti e con l'esperienza dei singoli, generando veri e propri schemi narrativi mentali. Secondo Victor Raskin (1985, 58-59 ss.), massimo esponente degli Humor Studies, questi schemi narrativi costituiscono l'impalcatura strutturale delle barzellette e possono opportunamente essere definiti script. Questo termine è più ampio e complesso di «stereotipo», in quanto condensa l'accezione informatica di «sequenza ordinata di istruzioni»e quella cinematografica di «sceneggiatura». La duplice accezione rende script il lessema ideale per spiegare la strutturazione della barzelletta. Uno script, per usare le parole di Raskin (ivi, 81), è:

[...] a large chunk of semantic information surrounding the word or evocated by it $[\ldots]$ a cognitive structure internalized by the native speaker and it represents the native speaker's knowledge of a small part of the world. 


\section{Tipologia, STRUTTURA E FUNZIONI DELLA BARZELLETTA}

\subsection{La sovrapposizione di script}

In Semantic Mecbanisms of Humor (a tutt'oggi il massimo contributo allo studio delle barzellette), Raskin (1985) rielabora le principali teorie filosofiche e semiotiche sul rapporto tra bumour e «linguaggio» per giungere a proporre le condizioni necessarie perché un testo narrativo sia comico:

- a presupposition is shared by $\mathrm{S}$ [peaker] and $\mathrm{H}$ [earer]

- an implicature is produced by S[peaker]

- a possible world is evoked by the text

- a speech act occurs. (ivi, 56) ${ }^{6}$

Lo studioso rileva, tuttavia, che queste condizioni, pur necessarie, non sono sufficienti. Utilizzando il concetto di script, che esprime la relazione tra «implicatura» e «atto linguistico», Raskin formula la sua Main Hypothesis riducendo a due le condizioni necessarie e sufficienti:

A text can be characterized as a single-joke-carrying if both the conditions [...] are satisfied:

I) the text is compatible, fully or in part, with two different scripts;

II) the two scripts with which the text is compatible are opposite.

The two scripts with which some text is compatible are said to overlap fully or in part on this text [...] therefore, the set of two conditions [...] is proposed as necessary and sufficient conditions for a text to be funny. (ivi, 99 ; corsivo mio) ${ }^{7}$

Il modello di Raskin può essere esemplificato, applicandolo a due barzellette russe sugli italiani del corpus esaminato in questo lavoro:

[1] Если у итальянцев национальное блюдо - пицца, то у русских - напицца ${ }^{8}$

$$
1^{\circ} \text { script: }
$$

gli italiani mangiano sempre pizza

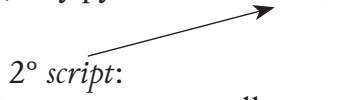

i russi bevono sempre vodka

sourapposizione $\rightarrow$ PIZZA [nuщua] [NA]PIZZA [напиться]

6 Raskin utilizza i termini implicature («implicatura») e speech act («atto linguistico») come oggi comunemente utilizzati in filosofia del linguaggio, riferendosi, cioè, alle rispettive teorie di H. Paul Grice e John Searle.

7 Il frammento qui citato è nel testo quasi interamente evidenziato in grassetto.

8 Se il piatto nazionale degli italiani è la pizza, quello dei russi è ubriacarsi [na-pit'sja; pronuncia: «napizza»]. 
[2] - Сима, ты посмотри какая кривая эта Пизанская башня!

- Моня, ты на себя посмотри! ${ }^{9}$

[1] contiene un gioco di parole, ma è più facile da comprendere per un italiano rispetto a [2]. In [1] sia le presupposizioni, sia le implicature sono infatti condivise da russi e italiani. In [2] solo uno dei due script è condiviso da italiani e russi: la torre di Pisa è storta (bislacca, penzolante). Il secondo script (opaco agli italiani) è complesso e «narra» che gli ebrei sono sempre bislacchi e malcombinati (storti), agli ebrei va sempre tutto storto, gli ebrei vedono tutto storto (criticano sempre tutto):

sourapposizione $\rightarrow$ storta (torre) $\leftrightarrow$ storto (Monja [= ebreo])

A un italiano che non conosca a fondo la cultura russa manca quella «conoscenza di una piccola parte del mondo» che, come si è visto, fa parte della «struttura interiorizzata» (Raskin 1985, 81) di un nativo russo: $\mathrm{Si}$ ma e Monja, infatti, sono tipici diminutivi di antroponimi particolarmente diffusi tra gli ebrei russi e quindi antonomastici, cioè stereotipici (come il nome «Gennarino» è stereotipo antonomastico tipico di «napoletano») ${ }^{10}$.

Gli esempi [1] e [2] indicano subito quella che risulterà un'anomalia del corpus delle barzellette russe «sugli italiani»: in buona percentuale, in realtà, non hanno affatto come bersaglio gli italiani, bensì i russi stessi (cf. Visani 2005, 158) o altri outgroup. Già in [1], a ben vedere, uno stereotipo inoffensivo sugli italiani (sul cibo) si sovrappone a uno meno inoffensivo sui russi (sull'alcolismo); in [2], il primo script non è uno stereotipo, ma un dato oggettivo (la torre è effettivamente storta), mentre il secondo script si fonda sullo stereotipo dell'ebreo (russo) storto: si tratta quindi di un testo che fa parte dell'immenso corpus di barzellette russe sugli ebrei, ideate e narrate dagli ebrei russi stessi (forse i massimi cultori dell'auto-derisione nella moderna tradizione delle «barzellette etniche») ${ }^{11}$.

9 - Sima, guarda com'è storta la torre di Pisa! - Monja, ma guardati tu!

10 Il primo deriva da Semen, il secondo o da Mojsej o, pure, da Semen.

11 Le barzellette sugli ebrei sono opportunamente definite «barzellette ebraiche»: sebbene esistano barzellette propriamente antisemite (ideate da non ebrei) e talvolta estremamente aggressive (cf. Raskin 1985, 212-214), la maggior parte hanno origine endogena e sono quindi un caso di «auto-derisione»; infatti, in numerosi casi, gli script utilizzati per il Jewish bumor sono noti solo (o soprattutto) all'ingroup e non sono compresi all'esterno (ivi, 212). Negli altri casi, le barzellette etniche sono sostanzialmente di origine esogena. 


\subsection{Le barzellette etniche}

Tra le barzellette registrate presso diversi popoli del mondo contemporaneo, quelle «etniche» sono le più «universali» e hanno particolare successo in tutte le culture (Davies 2005, 70); non sempre, tuttavia hanno a che vedere con stupidità, sporcizia o lentezza, ovvero, con quelli che Davies (ibidem) considera i pattern della derisione etnica ${ }^{12}$.

Tendenzialmente, osserva la studiosa (ivi, 75), l'ipotesi che le barzellette etniche abbiano come target un gruppo in conflitto con quello del narratore della barzelletta è falsificabile per due ragioni: da un lato, se si esaminano i gruppi oggetto di derisione e quelli che li deridono, non si riscontrano conflitti (anzi, nella maggior parte dei casi, si tratta di popoli verso cui c'è una bonaria indifferenza); dall'altro, se si considerano i gruppi realmente ostili, non si riscontrano casi di sistematica derisione etnica basata sulla stupidità (ai nemici veri non si attribuisce stupidità). Si deridono, secondo Davies (ibidem), solo gli outgroup più «deboli». In altre parole, ci sarebbe una differenza di status tra i gruppi che deridono e quelli che sono derisi; questi ultimi sarebbero più socialmente fragili o emarginati (ivi, 76). Come vedremo, questo vale soprattutto per le barzellette sulla «stupidità», ma non per quelle che evidenziano caricature (per esempio, prestanza sessuale e ricchezza) o difetti visti con condiscendenza (per esempio, l'incuranza della legge).

Secondo Raskin (1985, 180), gli script su cui si basano le barzellette etniche non fanno parte della conoscenza «linguistica», ma di quella «enciclopedica»; tuttavia, in realtà, le due cose sono collegate (cf. Salmon Kovarski 1995), tanto più che molte barzellette etniche sono basate sugli errori grammaticali o ortoepici dei membri dell'outgroup quando parlano la lingua dell'ingroup. Oltre agli script sulla «stupidità» (ivi, 185-191), l'«astuzia» (ivi, 191-194), la «superiorità nazionale» (ivi, 202-205), Raskin individua utilmente anche script etnici anomali o "pseudo-etnici» (ivi, 194200), che non rispondono ai seguenti parametri che determinano una barzelletta propriamente «etnica»:

12 Davies $(2005,72)$ riporta una tabella che elenca vari Paesi (europei ed extra-europei) con la relativa etnia derisa per «stupidità»: incredibilmente, non è menzionata la Russia, né alcun altro Paese slavo (e, tra i Paesi dell'Europa Orientale, c’è la sola Romania). Viceversa, polacchi e ucraini figurano tra i popoli derisi per «stupidità» (rispettivamente negli USA e in Canada). Inoltre, Davies (ibidem) abbina all'Italia la derisione per «stupidità» dei «meridionali» («Southern Italians») e non dei «carabinieri»: pur imparentate (come indica l'accento con cui parlano «appuntato» e «maresciallo»), le due categorie nella realtà non coincidono. 
- the joke is truly ethnic if and only if its main opposition or one of its main oppositions involves at least one truly ethnic script;

- if the joke is truly ethnic, the removal of the evoked ethnic script renders it incomprehensible;

- if the joke is truly ethnic, it treats the individuals involved in it solely and entirely as members of the targeted ethnic group;

- if the joke is truly ethnic, the targeted ethnic group may be substituted for only by another ethnic group which shares the evoked ethnic script with it. $\left(\right.$ ivi, 207) ${ }^{13}$

Raskin (ivi, 205) definisce «pseudo-etniche» le barzellette che violano la prima condizione fondamentale, quella di implicare almeno uno script propriamente etnico.

\section{TIPOLOGIA DEL CORPUS E ANALISI DEI TESTI}

\subsection{Il corpus}

Come anticipato, la natura squisitamente orale delle barzellette rende difficile reperire un corpus affidabile e rappresentativo e dimostrare con rigore quali siano le fonti primarie degli stereotipi su cui questi testi si formano. Per quanto concerne il corpus, si consideri che la narrazione spontanea di barzellette è correlata in modo piuttosto aleatorio ai contesti sociali: non si può prevedere quando, dove e chi racconterà una barzelletta, tanto meno su che cosa. La narrazione spontanea, infatti, è innescata bottom up, per congruenza tematica con temi che si presentano nella conversazione in modo più o meno casuale. Se, viceversa, si forza un parlante a raccontare barzellette, è probabile che non sia in grado di ricordarne e recitarne più di un paio (per motivi soggettivi difficilmente verificabili); inoltre, la richiesta esplicita del ricercatore (procedura top down) è un approccio poco affidabile per analisi statistiche e può indurre reticenza, soprattutto se si tratta di barzellette che denigrano l'outgroup a cui appartiene il soggetto interrogato o il ricercatore stesso. Per quanto riguarda la relazione tra testo ed effetto comico, tanto più nel caso delle barzellette russe, «ogni storiella scritta è solo un'eco sbiadita di quello che essa è sulla bocca di un narratore», e i narratori russi sono davvero speciali (Gandolfo 1991, 11).

13 Alle barzellette ebraiche Raskin dedica una sezione specifica (1985, 209-221), ma si limita a menzionare, senza affrontarlo, il tema dell'auto-derisione. 
Per quanto riguarda le barzellette etniche russe, osserva Visani (2005, 157-158), è opportuno distinguere tra quelle propriamente «etniche», quelle sulle minoranze ben note che convivono (o convivevano) con i russi nello stesso spazio nazionale, e quelle cross-cultural, riferite a veri «stranieri» di cui i russi hanno scarsa conoscenza, ereditando la rappresentazione stereotipica comune a numerosi Paesi europei ${ }^{14}$.

Le barzellette russe sugli italiani sembrano situarsi al confine tra le due categorie, nutrite di stereotipia autoctona e di stereotipi interculturali. Per studiarle, e acquisire quindi informazioni sui cliché che i russi associano a questi «stranieri» particolarmente amati e affini, non esiste un corpus scritto alternativo a Internet: non solo non risultano pubblicate raccolte di siffatti testi, ma questo «filone» - rispetto a quello sui čukči o sugli estoni (protagonisti delle barzellette russe su «stupidità»e «lentezza») - è quantitativamente esiguo. L'opzione del corpus elettronico è un buon compromesso per analizzare i testi in forma scritta, ma in un ambiente quasi-spontaneo: il format dialogico di blog e chat, ad esempio, consente ai singoli utenti di inserire direttamente i propri testi (dopo essersi iscritti) e in modalità di semi-anonimato (mediante un nickname spesso opaco).

Il «filone italiano», comparato ad altri filoni etnici, non è quantitativamente rilevante, ma sul piano qualitativo consente di individuare gli stereotipi sugli italiani più diffusi tra i russi e di interrogarsi sulla tipologia e cronologia delle loro fonti. Non si dimentichi, infatti, che solo gli stereotipi condivisi fanno ridere (anche un ascoltatore che non condividesse i pregiudizi può comprendere la barzelletta solo se li riconosce). Ciò autorizza a ritenere che le barzellette condivise sui blog (pur spesso poco «divertenti») siano generate da script che i russi riconoscono come propri.

La ricerca del siti che hanno fornito il corpus è avvenuta principalmente mediante i motori google.it e yandex.ru - rispettivamente i più usati in Italia e Russia - e le parole chiave utilizzate (in russo) sono state: anekdoty ob ital'jancach («barzellette sugli italiani»), per quanto riguarda i testi delle barzellette, e ital'jancy glazami russkich («gli italiani agli occhi dei russi») per quanto riguarda i testi sul «carattere degli italiani». I siti che rispondevano a queste parole chiave, da cui è stato estratto il corpus, non sono numerosi in assoluto, ma sono tutti «interculturali», cioè "sui russi in Italia» $\mathrm{o}$ «sui russi che vogliono andare in Italia» e che, per lo più, parlano o capi-

14 La maggior parte, sostiene Visani $(2005,162)$, sarebbero sui francesi, gli americani, gli inglesi e, più raramente, i tedeschi; tuttavia, la studiosa non offre dati in proposito (né sui criteri di selezione del corpus, né sui «siti internet» cui si rifà, né, infine, sui «russi» tra cui ha raccolto le barzellette $[i v i, 170])$. 
scono l'italiano (pur in modo approssimativo, come rivelano «traduzioni» e «traslitterazioni» reprensibili). I siti considerati nella ricerca sono russi, con l'eccezione di un solo sito ucraino in lingua russa (Tocbka.net), che (nella sezione putešestvija o travel) confronta i difetti di «italiani» e «ucraini», rivelando che i difetti che gli ucraini attribuiscono a se stessi sono i difetti che attribuiscono a se stessi i russi (cambiando la parola «ucraini» con «russi» il testo funziona in modo del tutto analogo). Si tratta nel complesso di testi che, in forma di barzelletta o di commento divulgativo, diffondono stereotipi già piuttosto radicati e noti.

\subsection{Barzellette pseudo-etniche o su altri gruppi etnici (non gli «italiani»)}

Poiché, come anticipato, nel corpus si incontra una buona percentuale di testi che in realtà non sono barzellette russe sugli italiani, le barzellette presentate come anekdoty ob ital'jancach si dividono in due tipologie:

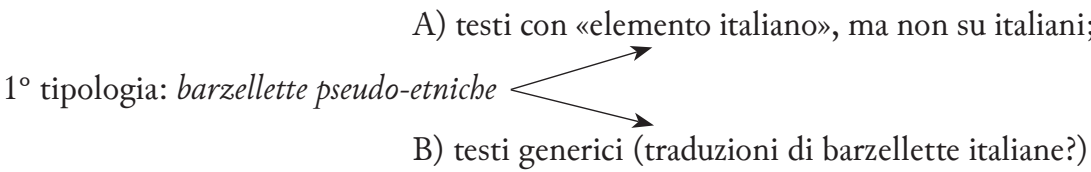

In A) e B) la confusione di compilatori e contributori dei siti analizzati è dovuta al fatto che, nelle barzellette, vengono menzionati l'Italia, gli italiani e/o nomi italiani, sebbene questi elementi siano irrilevanti rispetto alla vera opposizione degli script, in realtà generici e/o universali (Raskin 1985, 206207) ${ }^{15}$. Le differenze tra A) e B) sono sfumate. In alcuni casi, il sito stesso (ad esempio, Ice-nut) li include esplicitamente in una sezione di «barzellette italiane» tradotte in russo. In altri casi, invece, l'origine italiana è stabilita a orecchio. Ad esempio, è riconoscibile il rifacimento di una barzelletta in dialetto veneto che deride i veneziani (lenti, pigri e illogici), così presentata nel sito:

[3] Лето, квартира на пятом этаже, открытый балкон. Жена гладит бельё, муж на диване читает газету, маленький сын бегает во дворе. Вдруг муж начинает громко зевать. Жена: “Джузеппе, позови сына домой, всё равно у тебя рот открыт”. (Ice-nut) ${ }^{16}$

15 In altre parole, la presenza di «Pierino» non rende una barzelletta etnica: infatti, l'antonomasia «Pierino» può essere sostituita con l'analogo di qualsiasi altra cultura, ad esempio con Vovočka (il «Pierino» russo).

16 Qui e in seguito la traduzione dal russo delle barzellette è mia: Estate, un appartamento al quarto piano, la finestra del balcone è aperta. La moglie stira, il marito legge il giornale sul divano, il bambino gioca in cortile. A un tratto il marito si mette rumorosamente a 
Anche in altri casi, parrebbe quasi certo trattarsi di traduzioni di testi italiani:

[4] Ребенок никак не хочет заснуть. Итальянка говорит мужу: - Может, мне ему что-нибудь спеть? - Ну, зачем же так сразу. Попробуй с ним сначала по-хорошему. (Italija po-russki) ${ }^{17}$

[5] Маленький Джанни присутствует на суде, где разводят его родителей. Судья спрашивает: - Скажи, с кем бы ты хотел жить - с папой или с мамой? - Зависит оттого, - отвечает малыш, - кому достанется компьютер. $(\text { Ice-nut })^{18}$

[6] Уезжает муж в Италию, жена просит - Ваня купи мне бюстгальтер. - А зачем тебе; у тебя же ничего там нет. - Ну я же тебе то трусы покупаю. $(\operatorname{RimIV})^{19}$

[7] Осама бен Ладен на экскурсии в Италии, осматривает достопримечательности. Дойдя до Пизанской башни, усмехаясь, произносит: - Дилетанты! $(\text { Italija })^{20}$

[8] Встречаются две голливудские актрисы, одна восклицает: - Как ты хорошо выглядишь! - Да, я недавно вышла замуж за итальянца, такой потрясающий мужчина! Теперь только за итальянцев буду выходить. $\left(\right.$ anekdoty.ru) ${ }^{21}$

In [4] è derisa una moglie (qualsiasi) che non sa cantare, in [5] l'opportunismo di un figlio (qualsiasi), in [6] la poca virilità di un marito (qualsiasi), in [7] il primitivismo di Bin Laden. In [8], infine, anche se si fa effettivamente riferimento allo stereotipo degli «italiani» attraenti e passionali, non è quello uno dei due script su cui si fonda la barzelletta; applicando il criterio di Raskin, se si cambiano gli italiani con i francesi (che ancor più

sbadigliare. La moglie: «Giuseppe, visto che hai la bocca aperta, chiama tuo figlio e digli che venga a casa». La versione (in dialetto veneto) mi è nota fin da bambina: «Maria, dato che hai la bocca aperta, chiama anche mio figlio».

17 Non si riesce in alcun modo a far dormire il bambino. L'italiana dice al marito: - Magari gli canto qualcosa? - Beh, non è il caso di usare le maniere forti, prova prima con le buone.

18 Il piccolo Gianni presenzia all'udienza per la separazione dei genitori. Il giudice gli chiede: - Dimmi, con chi vuoi andare, con papà o con mamma? - Dipende, - risponde il piccolo, - a chi va il computer.

19 Il marito parte per l'Italia, la moglie gli chiede - Vanja, comprami un reggiseno. Che te ne fai, tanto lì non hai niente. - Beh, io però te le compro le mutande.

20 Osama bin Laden è in gita in Italia, ammira i monumenti. Quando arriva alla torre di Pisa, con una risatina sprezzante dice: - Dilettanti!

21 Due attrici di Hollywood si incontrano. Una esclama: - Ma come stai bene! - In effetti, di recente ho sposato un italiano, un uomo eccezionale, d'ora in poi sposerò solo italiani. 
rispondono allo stereotipo della passionalità), il testo funziona lo stesso ${ }^{22}$. Il primo script di [8] è generico e duplice: ci si sposa una volta sola (se hai un buon marito, non ne cerchi un altro), ma una «cosa buona» si ripete; il secondo script non è etnico, se non nei limiti in cui riguarda le attrici di Hollywood (americane), che si sposano di continuo e qui risposerebbero più volte un italiano. Tuttavia, la stessa barzelletta, sullo stesso sito, è presente anche nella versione «si incontrano due amiche facoltose», dove appare chiaro che il target della barzelletta sono le donne frivole e capricciose in generale: lo stereotipo sugli italiani è irrilevante.

C) testi che in realtà deridono i russi o altre etnie (non «gli italiani») ${ }^{23}$ $2^{\circ}$ tipologia: barzellette etniche

D) testi che effettivamente deridono «gli italiani»

Il gruppo C), oltre a [2], comprende, ad esempio:

[9] 90\% процентов россиян живут в стрессе. Остальные десять процентов живут в Англии, Италии и других благополучных странах. $(\operatorname{RimIV})^{24}$

[10] Новый русский пишет из Италии знакомому: "Высылаю фото, это я в музее рядом с Аполлоном. Который в шортах, это я”. (Italija) ${ }^{25}$

[10] è raccontata anche nelle versioni «un uomo scrive dall'Italia» (RimIV) e «un ebreo scrive dall'Italia» (ibidem) entrambe con la variante «quello nudo è Apollo»;

[11] Итальянская и русская тюрьмы решили на месяц поменяться заключенными. Через три дня русские попросили пожизненного заключения, а итальянцы - немедленной смертной казни. $(\text { Ice-nut })^{26}$

22 Essendo molto lunghe, le barzellette da cui si può evincere che i francesi, per i russi, sono amanti passionali, non sono qui riportate; sono reperibili sui siti RimIV, Italija porusski, Vysokovskij.ru.

23 Visani $(2005,170-171)$ rileva che spesso, nelle barzellette russe cross-cultural, i russi figurano in una posizione di inferiorità morale e intellettuale (sono rozzi e ubriaconi), nonché di svantaggio materiale; tuttavia, avendo l'ultima parola nella barzelletta, manifestano la propria genialità contrapposta alla prevedibile ordinarietà degli «stranieri».

24 Il 90\% dei russi vive in ansia. Il restante 10\% vive in Inghilterra, Italia o altri Paesi prosperi.

25 Un «nuovo russo» scrive a un amico dall'Italia: «Ti mando una foto: sono al museo con Apollo, quello in calzoncini corti sono io».

26 Un carcere italiano e uno russo hanno deciso di scambiarsi per un mese i detenuti. Dopo tre giorni, i russi hanno pregato di avere l'ergastolo e gli italiani la pena di morte immediata. 
In [11] si vede che il primo script riguarda il lassismo delle carceri italiane (stereotipo: gli italiani non applicano la legge e amano «spassarsela» sempre e dovunque); ma è il secondo script - basato sullo stereotipo (negativo) delle prigioni russe $-\mathrm{a}$ innescare la risata.

In C) rientrano anche barzellette che sono propriamente «multi-etniche», poiché coniugano stereotipi riferiti a più popoli. Alcune sono chiaramente russe (il climax è innescato da script dei russi su se stessi) e riflettono almeno uno stereotipo sugli italiani:

[12] Англичанин: 'Мой дом - моя крепость', Американец: 'Мой автомобиль' моя крепость', Итальянец: 'Моя семья - моя крепость', Русский: 'Моя крепость - 40 градусов'. (anekdoty.ru) ${ }^{27}$

In [12] sono espressi stereotipi su vari popoli, compreso l'attaccamento degli «italiani» alla famiglia, ma la risata è innescata dalla sovrapposizione di krepkij («forte») con krepost' («fortezza») che deride la passione russa per la «fortezza» (alcolica) della vodka.

\subsection{Stereotipi e barzellette etniche sugli italiani}

Per meglio classificare le barzellette propriamente etniche sugli «italiani» (sotto-categoria D) e i relativi script, è opportuno soffermarsi brevemente su alcuni testi descrittivi sul «carattere degli italiani» (talvolta accessibili sullo stesso sito dove sono raccolte le barzellette). L'esempio più indicativo è dato dalla voce anonima Italjanskij charakter («Il carattere italiano») del sito Tut vsja Italija, che si prefigge lo scopo di «comprendere almeno un poco cosa mai sia il carattere italiano». L'anonimo autore propone dettagliatamente alcuni tra i più significativi stereotipi sugli italiani, alcuni radicati tra i russi, ad esempio:

- sono un singolo popolo solo per gli stranieri; in ogni regione hanno abitudini, usi e tradizioni diverse;

- il $90 \%$ predilige la cucina italiana;

- sono fissati col calcio;

- sono fissati col vestiario e sono leziosi;

- la famiglia è la cosa più importante e si resta con la mamma fino a 35 anni;

- leggono poco (la loro stessa vita è un'avventura).

27 L'inglese: «La mia casa è la mia fortezza»; l'americano: «La mia macchina è la mia fortezza»; l'italiano: «La mia famiglia è la mia fortezza»; il russo: «La mia fortezza sono 40 gradi». 
A questo punto, l'autore rimanda esplicitamente a un testo-chiave della stereotipia sugli italiani. Si tratta della traduzione russa del testo inglese di Martin Solly, Xenophobe's Guide to the Italians del 1995 (cf. Solly 1999) ${ }^{28}$. In sintesi, dai brani citati sul sito emergono i seguenti stereotipi ${ }^{29}$ :

Gli italiani recitano tutta la vita come fossero in una «lunghissima commedia», parlano e si muovono secondo un «copione» che hanno appreso fin da piccoli. (ivi, 14)

Gli italiani sono per loro natura imbroglioni e si trovano sempre persone disposte a tutto per facili guadagni. (ivi, 64)

Le leggi sarebbero perfette se non ci fossero gli italiani che delle leggi se ne fregano altamente. (ivi, 66)

Sul sito TurGid.ru, la pagina dedicata all'Italia viene aperta da un articolo di un'«esperta» («Gli italiani. Cosa sappiamo di loro»), in cui gli «italiani» sono definiti «espansivi», «socievoli», «irascibili», «passionali», «disorganizzati», «sconclusionati», «modaioli», «passionali».

Nel complesso, nei commenti descrittivi dominano i seguenti stereotipi sugli «italiani» che si ritrovano puntualmente nelle barzellette propriamente «etniche» della tipologia D:

- sono emozionali, chiassosi, gesticolatori, litigiosi

[13] Группа итальянцев на экскурсии у каскада Ниагары. Гид говорит: - Если вы все сразу замолчите, то тогда сможете услышать, как шумит каскад. $(\operatorname{RimIV})^{30}$

[14] В городских автобусах над головой водителя всегда висит объявление. В Германии: “Строго воспрещается разговаривать с водителем”. В Англии:

28 La stessa collana comprende una serie di analoghi libretti su decine di altre popolazioni, dagli australiani ai canadesi, dai gallesi ai giapponesi. In russo questi libri sono stati tradotti con una modifica «politicamente corretta» del titolo: la parola «xenofobo» è stata bandita e sostituita dalla formula Eti strannye $X$ («Questi strani X»). Tra questi libri, inoltre, è interessante il volumetto sui russi di Elisabeth Roberts (uscito nel 1994 a Londra e in traduzione italiana nello stesso anno). Qui Roberts sintetizza anche quello che i russi «sospettano degli italiani» (ivi, 11), ovvero che siano «un popolo approssimativo, indolente, poco incline a rispettare le leggi e a mantenere gli impegni» (e subito aggiunge: «di qui l'istintiva, naturale e profonda simpatia che hanno per noi»).

29 I brani sono tratti, parrebbe, non dal libro, ma dalle citazioni riportate sul sito http://www.koob.ru/solly/, effettivamente presenti nel libro (le pagine tra parentesi sono state qui aggiunte).

30 Un gruppo di italiani alle cascate del Niagara. La guida dice: - Se state tutti insieme zitti per un momento, potrete sentire il rumore della cascata. 
“Будет лучше не разговаривать с шофером”. В Израиле: “Нет никакого интереса разговаривать с шофером”. В Италии: “Запрещено отвечать шоферу". (RimIV) $)^{31}$

Sui vari siti si trovano altre varianti di [14], ad esempio: «È vietato parlare col conducente! Gli servono le mani [per guidare]» (Čao kakao).

[15] За обедом в итальянской семье происходит отчаянная ссора, летят тарелки, перевертывается стол. Соседи дрожат от страха. Супруга собирает чемоданы и уходит. Но через два часа снова поднимается по лестнице и, толкнув дверь ногой, произносит: - Можешь считать, что тебе повезло. Моя мама тоже крепко поругалась с отцом и ушла к своей маме... (Italija po-russkij) ${ }^{32}$

- sono sesso-dipendenti, incostanti, infedeli e prolifici

[16] Аптека в Италии в день всех влюбленных: - Здравствуйте! - Закончились! (Italija po-russkij) ${ }^{33}$

Sul sito TurGid vengono forniti dettagli non solo sulla «passionalità» degli italiani, ma sulla disposizione a parlare pubblicamente di sesso e a praticarlo fino alla vecchiaia: «Del resto, che dire, il sesso è parte della vita e gli italiani sono notoriamente pieni di vita», scrive l'anonimo autore. Nel forum del sito Neapol' po-slavjanski, in un messaggio («Iska», 02.07.2011) si legge che «la passione tipica degli italiani indica l'incostanza del loro amore, sebbene siano fedeli alla famiglia»; la frase è preceduta da quella che l'autore («Iska») definisce come sua «barzelletta italiana preferita»:

[17] Я так же влюблен в Лауру, как и в первый день нашей встречи! - И давно ты с ней знаком? - Уже два дня. ${ }^{34}$

[18] Итальянец и американец встречаются в баре. Американец говорит: - Если ты мне дашь молоток, то я тебе произведу большой корабль. Тогда ита-

31 Negli autobus pubblici sopra la testa del conducente c'è un avviso. In Germania: «È severamente vietato parlare col conducente». In Inghilterra: «È meglio non parlare col conducente». In Israele: «Non c’è alcun vantaggio a parlare col conducente». In Italia: «Non rispondere al conducente».

32 A tavola in una famiglia italiana è in corso uno spaventoso litigio, volano i piatti, viene ribaltato il tavolo. I vicini tremano di paura. La moglie fa le valige e se ne va. Ma dopo un paio d'ore sale le scale e, aprendo la porta con un calcio, dice: - Considerati fortunato, anche mia madre ha litigato ben bene con mio padre e se n'è andata da sua madre...

33 Una farmacia italiana nel giorno di San Valentino: - Buon giorno! - Finiti!

34 «Sono innamorato di Laura come il primo giorno che l'ho incontrata!», «E quando l'hai incontrata?». «Sono ormai due giorni». 
льянец отвечает: - А если ты мне дашь твою жену, то я тебе произведу

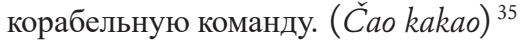

[19] Неаполь. Многодетная мамаша даёт интервью: - У меня 10 детей и всех их я назвала Дженнарино. - А как Вы их различаете? - Спрашивает журналист. - Я их зову по фамилии! (Italija po-russkij)

- sono mafiosi, banditi, disonesti (disprezzano la legge, non pagano le tasse)

[20] Сицилиец видит священника три раза в жизни: когда его крестят, когда он женится и когда его убивают. (С̆ao kakao) ${ }^{36}$

[21] Итальянцы и евреи имеют одну общую точку соприкосновения - банк. Одни им владеют, другие его грабят. (Čao kakao) ${ }^{37}$

[22] Сколько сицилийцев требуется, чтобы поменять лампочку? - Три. Один, чтобы поменял и два, чтобы убрали всех свидетелей. (Čao kakao) ${ }^{38}$

[23] Итальянская налоговая инспекция разработала новый эффективный метод оплаты налогов. Инструкция по оплате содержит только два пункта:

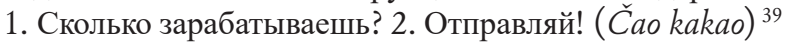

\section{- sono irresponsabili, superficiali}

[24] Что такое счастье по-итальянски? Это крепкое здоровье и... плохая память. (Ice-nut) ${ }^{40}$

- sono conservatori (più che mai nel cibo)

[25] Почему итальянцы не едят шашлык? - А ты пробовал спагетти на шампуры нанизывать?! $(\operatorname{Rim} I V)^{41}$

35 Un italiano e un americano si incontrano in un locale. L'americano dice: - Se mi dai un martello, ti faccio una grossa nave. L'italiano risponde: - Se mi dai tua moglie, ti faccio tutto l'equipaggio.

36 Un siciliano vede il prete tre volte nella sua vita: quando lo battezzano, quando si sposa e quando lo seppelliscono.

37 Gli italiani e gli ebrei hanno una cosa in comune: le banche. Gli uni le possiedono, gli altri le rapinano.

38 Quanti siciliani servono per cambiare una lampadina? - Tre. Uno per cambiarla e due per eliminare tutti i testimoni.

39 L'agenzia delle entrate ha elaborato un nuovo metodo per pagare le tasse. Il modulo di pagamento contiene solo due punti: 1. Quanto guadagni? 2. Paga!

40 Cos'è la felicità all'italiana? È buona salute e... cattiva memoria. ghetti?

41 Perché gli italiani non mangiano gli spiedini? - Hai provato ad avvolgerci gli spa- 
[26] Сколько итальянцев нужно, чтобы поменять лампочку? Двое. Один закручивает лампочку, а другой посыпает её тёртым сыром Пармезаном. (Саo kakao $)^{42}$

- sono ossessionati dal calcio

[27] Двое мафиози в джакузи. - У тебя охрана сколько человек? - Одиннадцать. А у тебя? - И у меня. 11 - Слушай, может в футбол сыграем? (Ice-nut) ${ }^{43}$

Alcune barzellette uniscono due o più dei suddetti stereotipi:

[28] Римский таксист объяснял туристу, что только НАСТОЯЩИЕ мужчины могут водить такси в столице Италии: - Мы используем левую руку, чтобы подавать сигнал, а правой рукой мы приветствуем женщин... - Но как же вы ведете машину? - удивленно перервал его турист. - Но я же вам только что сказал, что лишь НАСТОЯЩИЕ мужчины могут водить такси в Риме! (Italija) ${ }^{44}$

Che [28] sia una barzelletta russa è evidente: infatti, l'espressione nastojaščij mužčina («vero uomo») in italiano è usata solo in senso (auto-)ironico, mentre in russo è usata solo in senso letterale (cf. Salmon 1998, 457) ${ }^{45}$.

Sub specie di barzellette da alcuni siti vengono anche riportate citazioni divertenti di personaggi (italiani e non) che iterano stereotipi positivi e negativi sugli italiani:

[29] “Швейцарская столица Берн в пять раз больше, чем кладбище в Неаполе, но на кладбище в Неаполе в пять раз веселее, чем в швейцарской столице Берн”. Luciano de Crescenzo, итальянский писатель. (Italija) ${ }^{46}$

[30] “У итальянцев в голове только две мысли, последняя - это спагетти”. Саtherine Deneuve, французская киноактриса. (Italija) ${ }^{47}$

42 Quanti italiani ci vogliono per cambiare una lampadina? Due. Uno l'avvita e l'altro ci mette sopra il parmigiano.

43 Due mafiosi nell'idromassaggio. - Quanti ragazzi ha nella tua guardia del corpo? Undici, e tu? - Anch'io 11. - E che ne dici di fare un incontro di calcio?

44 Un tassista romano spiega al turista che solo un VERO uomo può guidare il taxi nella capitale italiana: - Noi usiamo la mano sinistra per suonare il clacson e la destra per salutare le donne... - E la macchina come la guidate? - lo interrompe il turista stupito. - Ma le ho appena detto che solo un VERO uomo può guidare la macchina a Roma!

45 La fissazione per il «vero maschio» è alla base dell'autentica passione per l'italiano vero» di Toto Cotugno, da una canzone (del 1983) che ha spopolato in URSS per anni.

46 «La capitale svizzera Berna è cinque volte più grande del cimitero di Napoli, ma il cimitero di Napoli è un luogo cinque volte più divertente della capitale svizzera Berna». Luciano De Crescenzo, scrittore italiano.

47 «Gli italiani hanno in testa due sole cose, la seconda sono gli spaghetti». Catherine Deneuve, attrice francese. 
Infine, non mancano nel corpus riferimenti alle più tragiche pagine di cronaca italiana:

[31] Капитан итальянского лайнера кадрит пассажирку: - Вы фильм "Гибель Титаника" видели? - Нет, не видела... - Щас покажу! (RimIV) ${ }^{48}$

Si può aggiungere che, al di fuori del corpus considerato, vi sono vari siti russi che propongono barzellette su Silvio Berlusconi (inserendo, ad esempio, le parole chiave ankedoty o Berluskoni) che, in buona parte, non sono traduzioni di testi italiani.

\section{LE FONTI DEGLI STEREOTIPI RUSSI SUGLI ITALIANI}

\subsection{Cinema italiano, russo e co-produzioni}

In generale, risalire alle fonti degli stereotipi etnici e all'epoca in cui si sono radicati può essere difficile. Come noto, la cultura italiana ha avuto ampia influenza su quella russa per quanto riguarda soprattutto l'architettura, la musica, il teatro e la letteratura ${ }^{49}$. La più evidente testimonianza di questo influsso culturale è testimoniata, nell'arco degli ultimi tre secoli, dalla crescente diffusione in lingua russa degli italianismi, che sono aumentati esponenzialmente negli ultimi decenni (cf. Denisova 2012, 97-104). In particolare, in epoca post-sovietica, soprattutto nella ricezione dei «nuovi russi», l'immagine dell'Italia è associata allo stereotipo della (supposta) «bella vita italiana», alle più prestigiose località di villeggiatura italiane, all'alta moda e alla «esotica» (per i russi) cucina del nostro Paese (ivi, 118-119). Tuttavia, si tratta di un'immagine sostanzialmente «artificiale» (ivi, 101-102); infatti, come indicano le barzellette e i commenti sugli «italiani», a condizionare gli stereotipi dei russi nell'arco del Novecento non è stato il troppo recente ed esclusivo made in Italy, bensì l'immagine popolare degli «italiani» stratificatasi in epoca sovietica attraverso la cultura trasversale di cui poteva usufruire il popolo sovietico nel suo complesso. Poiché i cittadini sovietici per lo più non potevano andare all'estero, né entrare liberamente in con-

48 Il comandante di una nave da crociera italiana tacchina una passeggera: - L'ha visto «Titanic»? - No, non l'ho visto... - Dai che te lo mostro!

49 Per una rassegna sulla presenza degli italiani e della cultura italiana nella storia russa, cf. Bautdinov 1986. 
tatto con i pochi occidentali che soggiornavano in URSS, le immagini che i sovietici collegavano all'Italia erano per lo più fornite (e deliberatamente calibrate dalla censura) attraverso due canali principali: la letteratura per l'infanzia e i testi audiovisivi, in primis il cinema ${ }^{50}$.

Nonostante la passione delle masse sovietiche per le canzoni italiane (Celentano, Albano e Romina, i Ricchi e Poveri, Toto Cotugno ecc.), nel caso degli stereotipi sugli «italiani» che emergono dalle barzellette e dai commenti del corpus analizzato, meritano particolare attenzione alcune specifiche fonti cinematografiche che, almeno in buona parte, hanno profondamente condizionato in epoca sovietica l'immagine che ancora oggi i russi hanno dell'«Italia» e degli «italiani».

Nel periodo 1945-1970 è stato il cinema italiano a innescare la modalità in cui i russi hanno categorizzato gli «italiani» e la (supposta) vita nel nostro Paese. L'influsso sulla cultura sovietica dei film genericamente attribuiti al «neorealismo» italiano è attestato ancora oggi (cf. Žukova 2014) ${ }^{51}$. Film come, ad esempio, Roma città aperta (1945) e Ladri di biciclette (1948) hanno contribuito a forgiare gli stereotipi sulla famiglia, la prolificità, la meridionalità, la passionalità, la disonestà degli italiani; espressioni come «dolce vita» e «paparazzi» sono entrate in russo dal cult-movie di Federico Fellini La dolce vita (1960), nutrendo gli stereotipi sulla vitalità, vivacità, predisposizione alle avventure amorose, passionalità e teatralità degli italiani. Anna Magnani, Sofia Loren e Marcello Mastroianni sono divenuti per due generazioni di russi i prototipi dell'italianità. Per quanto riguarda l'associazione tra Italia e mafia, di grande impatto è stata in Unione Sovietica, nella seconda metà degli anni Ottanta, la trasmissione del telefilm a puntate (co-produzione internazionale) La piovra (Sprut), seguito con vero e proprio fanatismo, come può ricordare chiunque vivesse in URSS in quegli anni ${ }^{52}$.

Questa prima fase di intenso contatto con le immagini dell'Italia cinematografica ha posto le basi perché il cinema sovietico stesso iterasse quegli

50 I bambini sovietici leggevano senza eccezione Gianni Rodari (in particolare Le avventure di Cipollino) e Pinocchio in celebri traduzioni russe curate da scrittori della statura di Samuil Maršak e Aleksej Tolstoj.

51 Il cinema italiano ha creato le premesse per uno straordinario interesse per l'Italia e la sua cultura almeno fino al crollo dell'URSS, quando il mercato post-sovietico è stato invaso da prodotti nord- e sudamericani.

52 Le puntate successive, tanto più dopo il crollo dell'URSS, hanno visto lentamente decrescere la popolarità del telefilm, anche a causa della morte del protagonista, il commissario Cattani, impersonato da Michele Placido, altra icona di rilievo nell'immaginario russo sugli «italiani». 
stessi stereotipi sugli italiani, diffondendoli in modo capillare fino ai giorni nostri. In particolare, sono stati tre cult-movies, dagli anni Settanta agli anni Novanta, ad aver esercitato una fascinazione senza precedenti su almeno due generazioni di russi, rafforzando gli stereotipi su passionalità, leggerezza, disonestà, illegalità, simpatia, rumorosità ed espressività gestuale.

Il primo film è la nota commedia Neverojatnye priključenija italjancev $v$ Rossii, del 1973, co-produzione italo-sovietica (uscita l'anno seguente in Italia col titolo Una matta, matta, matta corsa in Russia) ${ }^{53}$. Qui vengono ribaditi gli stereotipi sugli italiani intraprendenti e sensibili ai facili guadagni, nonché prolifici e sanguigni. Tra le citazioni più note, la frase di Rosario, informato che gli è nata l'ottava figlia femmina:

У вас в Риме родилась девочка, поздравляем! [...] - Восьмую родила, кто их возьмет в жёны! ${ }^{54}$

Tuttavia un ruolo ben più cruciale lo hanno avuto altre due opere. La prima contiene la celeberrima canzone $M y$ bandito, gangsterito (musica di G.I. Firtič, testo di E.P. Čepoveckij), un capolavoro in pseudo-italiano ${ }^{55}$. La canzone fa parte della $4^{a}$ puntata del cartone animato televisivo Priključenija kapitana Vrungelja (Le avventure del capitano Vrungel'), realizzato in 13 puntate tra il 1976 e il 1979, e trasmesso a partire dal gennaio 1980. Liberamente ispirato all'omonimo racconto umoristico dello scrittore sovietico Andrej Nekrasov (pubblicato per la prima volta nel 1937), il cartone, realizzato a Kiev, è ricco di spunti parodici (da Stalin a James Bond). Tra i protagonisti figurano i mafiosi italiani Džuliko Banditto e De Lja Voro Gangsteritto ${ }^{56}$, interpretati dalle voci degli attori Semen Farada e Aleksandr Burmistrov. La canzone è rimasta nella memoria di chiunque abbia vissuto in URSS negli anni Settanta-Ottanta, e il celeberrimo cartone ha esercitato un'influenza senza confronti su milioni di russi fino a tempi

53 I dati forniti in Utevskij 1996, relativi alla posizione di leader del noleggio e della vendita dei film, confermano che Neverojatnye priključenija ... ha avuto più di 49 milioni di spettatori (ivi, 277), secondo il sito CCCP-USSR (http://ussr-kruto. ru/2012/10/30/filmneveroyatnye-priklyucheniya-italyancev-v-rossii/), più di 50 milioni, condizionando il mito degli italiani žulikovatye («imbroglioncelli»).

54 A Roma le è nata una bambina. Congratulazioni! [...] Mi ha partorito l'ottava femmina, chi se le sposa tutte?

55 La modalità dello pseudo-italiano era già stata sperimentata nella celebre battuta del cult-movie sovietico Brilliantovaja ruka (1969) obliko morale (da oblik, «immagine», e moral', «morale»), che tutti hanno creduto fosse «vero italiano».

56 I nomi richiamano le parole «truffatore», žulik, e «ladro», vor, adattate alla pseudomorfo-fonologia italiana. Džuliko, pur con ruolo minore, era già presente nel racconto di A. Nekrasov. 
recentissimi. Gli articoli sul film, a trentacinque anni dalla sua prima trasmissione, non si contano e attestano questo successo strepitoso di pubblico e critica, con particolare riferimento alla componente musicale:

La genialità di un film la stabilisce il tempo. E se passano i decenni e gli spettatori restano incollati allo schermo significa che è un capolavoro, e tra i capolavori c'è «Vrungel'». (Rylev 2015)

I cartoni sono davvero un potente strumento di condizionamento della psiche, soprattutto infantile [...] il verso della canzoncina [delle Avventure del capitano Vrungel'] «Comunque la chiamerete, con quel nome la barca salperà» è diventato un modo di dire del russo. E con la canzone «Money, money» la gente ci imparava l'inglese. Per non dire che la storia «commuovente» di «banditogangsterito» la conosceva ogni scolaro. (Grinevski 2014)

Le parole della canzone sono in rima e costruite su radici russe o «europee» (bandit, gangster, bank, ecc.) con suffissi che parodiano l'italiano con esplosivo effetto maccheronico (una stereotipica desinenza «o» è presente in tutte le parole russe o inventate):

Мы бандито-гангстерито,

Мы кастето-пистолето, о yes!

Мы стрелянто, убиванто,

Украданто то и это, о yes!

Банко-тресто-президенто

Ограблянто ун моменто, о yes!

И за энто режиссенто

Нас сниманто в киноленто, о yes!

Мы бандито, знаменито,

Мы стрелято пистолето, о yes!

Мы фиато разъежато

Целый день в кабриолето, о yes!

Постоянно пьем чинзано,

Постоянно сыто-пьяно, о yes!

Держим в банко миллионо

И плеванто на законо, о yes!

Мы пирато-гастролеро,

Мы синьоро де ля воро, о уes!

И гражданто убеганто
Siamo banditi-gangsteriti pugno-ferro-pistolo, o yes! Noi sparanto, ammazzanto, rubanto quest'e quello, o yes!

Presidento-banco-trust svaligianto un momento, o yes!

$\mathrm{E}$ intanto il registento ci filmanto in pellicolo, o yes!

Noi bandito, famosito, Noi sparanto pistoleto, o yes! Con Fiato girovato Tutto il di in cabrioleto, o yes! Tutto il dì beviam Cinzano, Tutto il dì sazio-brillo, o yes! ${ }^{57}$ Teniam in banco miliono E freganto di legge, o yes!

Noi pirato-gastrolero, Noi signoro del ladroro, o yes! E abitanto scappanto

57 L'effetto strepitoso del verso è dovuto all'omofonia tra la parola italiana «piano» entrata nel russo musicale e quella russa p’jan («ubriaco»). 
Врассыпанто престо скоро, о yes! А ля белля де бамбино Bce игранто в гангстерино, о yes! У мамано цап монето И стрелянто пистолето [...] presto ovunque tutto quanto

Alla bella de bambino, recitanto il gangsterino, o yes! A mamano, sgraf moneto, E sparanto pistoleto [...]

Alcuni versi della canzone sono diventati idiomatismi della lingua russa (Rylev 2015):

Il fascino incredibile di quest'opera in stile napoletano è dato dall'italiano maccheronico [...] che ha innescato istantaneamente la moda di scimmiottare l'italiano (e forse la successiva passione per la musica leggera italiana). Per quanto riguarda i versi «Tutto il dì beviam Cinzano, tutto il dì sazio-bevuto», sono diventati il simbolo della bella vita. Non c'è stato alcun intervento della censura sovietica: la canzone ha incantato tutti; del resto i sanguinari dettagli criminali del testo si potevano sempre spiegare con l'appartenenza dei banditi italiani al mondo agonizzante del capitalismo.

È interessante che, alcuni anni dopo, Semen Farada ha replicato l'esercizio canoro in pseudo-italiano. Nel film La formula dell'amore, questa volta con Aleksandr Abdulov, ha anche eseguito la composizione «Uno momento», pure divenuta un testo di culto.

Per quanto concerne la canzone «Bandito, gangsterito» [...] è entrata nel repertorio fisso dei programmi di cartoni animati e talvolta dei concerti per l'infanzia di capodanno. (Mažaev 2014)

Mažaev cita qui, per l'appunto, la terza fonte degli stereotipi russi, ovvero il cult-movie sovietico Formula ljubvi (La formula [magica] dell'amore, 1984), che ha come protagonisti dei truffatori travestiti da italiani (al seguito di un falso-Cagliostro) ${ }^{58}$. La canzone Uno momento (musica di G.I. Gladkov, testo di Ju.Č. Kim), cantata con accento russo in italiano maccheronico, ha avuto un successo tale, da aver indotto l'omonima catena di pizzerie della Russia post-sovietica a usare «Uno momento» come brand tipico dell'italianità:

Mare bella donna,

Che un bel canzone,

Sai che ti amo sempre amo

Donna bella mare.

Credere cantare

Dammi il momento

Che mi piace più.

58 Il film è ispirato al racconto di Aleksej Tolstoj Graf Kaliostro (Il conte Cagliostro; cf. Utevskij 1996, 474), pubblicato per la prima volta nel 1922 col titolo Lunnaja syrost' (Umidità lunare). 
Uno uno uno un momento,

Uno uno uno sentimento,

Uno uno uno complimento,

E sacramento sacramento sacramento.

Anche in questo caso, sono assemblate con intenzionale imprecisione le parole italiane più note ai russi (scegliendo come Leitmotiv il suffisso -mento), che danno tuttavia l'impressione che si tratti di italiano «vero» mediante un'apposita pseudo-lingua. I gesti e gli sguardi degli attori Semen Farada e Aleksandr Abdulov mentre cantano la «canzone napoletana» assecondano gli stereotipi degli italiani «artisti», «cantanti», «affascinanti seduttori»e «manipolatori».

A conferma dell'influsso del maccheronismo italiano sul linguaggio comico russo, si veda questa barzelletta sovietica compresa nel corpus:

[32] Один ответственный работник поехал по путевке в Италию. Вечером решил прогуляться по улицам Рима. Он еще дома был наслышан об итальянских женщинах. Стоит одна красавица у магазина ночных рубашек и подмаргивает ответственному работнику. Тот крутит головой. Она спрашивает: - Импотенто? Ответственный работник - от нее. - Трипперито? кричит красавица. Ответственный работник убегает на большой скорости и слышит вослед: - А! Замполито! $(\operatorname{Rim} I V)^{59}$

Un quarto film, questa volta interamente costruito sullo stereotipo dell'«italiana» (chiassosa, stravagante, capricciosa e seduttiva), è Moskovskie kanikuly (Vacanze moscovite), celebre melodramma grottesco del $1995^{60}$. Luciana, la protagonista italiana (che parla un italiano approssimativo con vistoso accento russo, come gli altri «italiani» del film) vuole seppellire il cane morto in Russia, patria di sua nonna (infatti lei parla bene russo, altra cosa poco credibile) ${ }^{61}$ è una caricatura dell'immagine della donna italiana emancipata, indipendente e ricca, consapevole della sua «italianità». Quando il commissario le chiede perché molčala (cioè, non aveva detto niente),

59 Un funzionario politico va in Italia. La sera decide di passeggiare per le strade di Roma. Già in Russia aveva sentito senza sosta parlare delle donne italiane. Vede una bella donna accanto a un negozio di intimo che gli fa l'occhiolino. Lui fa segno di no con la testa. Lei chiede: - Impotento? Il funzionario si allontana. - Tripperito? - urla la donna. Il funzionario corre più che può e sente - Ah, Zampolito! (Gioco di parole con le parole impotent, tripper [«scolo»] e zampolit [«vice-responsabile dell'attività politica»]).

60 Leader del noleggio dal luglio 1995 al gennaio 1996, solo in proiezione domestica il film ha avuto 749.000 spettatori (cf. Utevskij 1996, 257).

61 Dal punto di vista linguistico, come negli altri film, la verosimiglianza è vistosamente trascurata. 
Luciana risponde: «Zitta io? Nessuno mi aveva mai offeso così...». Anche in questo film viene utilizzata la tecnica del maccheronismo, in particolare sfruttando il suffisso italiano -issimo, strumento frequente di caricatura degli italiani (ad esempio, «spasibissimo», spasibo [«grazie»] + «issimo»).

\subsection{Voglio un marito italiano...}

Oltre ai film, altri testi riflettono gli stereotipi russi, sebbene, rispetto al cinema, siano da considerarsi non tanto delle «fonti», quanto degli echi di quelli stessi stereotipi. Merita almeno un cenno la recente narrativa sull'Italia al «femminile», che rispecchia nel suo insieme sia gli stereotipi sovietici più radicati, sia quelli di epoca post-sovietica. A titolo di esempio, si vedano i volumi di Marina Sorina (Voglio un marito italiano, 2006) e Natal'ja Osis (U samogo sinego morja. Ital'janskij dnevnik ${ }^{62}, 2011$ ). La pri$\mathrm{ma}$, originaria della provincia ucraina, scrive direttamente in italiano (scelta che peraltro penalizza lo stile sciatto del libro); la seconda autrice, che ha studiato teatro a Mosca ed è dottoranda in Italia, scrive in russo, optando per un linguaggio commerciale e uno stile aneddotico. Sorina e Osis vivono entrambe in Italia ed entrambe indicano o commentano alcuni cliché radicati tra i russi (in particolare, l'invidiabile - supposta - «bella vita» degli italiani e la loro fissazione per il cibo):

- E che cos'è l'Italia? - Non è una cosa... È un paese meraviglioso, dove ci sono mari e montagne, splende sempre il sole e non nevica mai. Per questo la gente è sempre allegra e ama cantare e ballare [...] qui ogni cosa è bella, anche la vecchiaia. È bello il cestino dell'immondizia, con tanto di stemma della città, bello il poliziotto in bicicletta, l'autobus arancione che si ferma davanti al bar [...]. Potevo toccare l'Italia, vivere l'Italia come un sogno che non passava, ma non ero un'italiana purtroppo. (Sorina 2006, 18, 83, 85) ${ }^{63}$

Dovrei dilungarmi ancora sulla cucina italiana, ma sul tema il Cibo in Italia tema sacro per gli italiani e inarrivabile per i non italiani - è stato scritto il bellissimo libro Il cibo è la felicità italiana (Eda - ital'janskoe sčast'e), e già il titolo mostra che non si può toccare di sfuggita una questione così importante [...] Fin da piccoli gli italiani sono abituati a riunirsi a tavola qualche minuto prima che la pasta sia pronta e con crescente tensione seguono come viene scolata, condita con la salsa, mescolata e, finalmente, portata in tavola... Suspense, co-

62 Dove il mare è più blu. Diario italiano.

63 I racconti di Sorina (2006) si occupano in parte di smascherare questi stereotipi, mettendoli pertanto in luce. 
me nei film hollywoodiani. Una procedura non solo emozionante, ma sacrale [...]. - Ma come ho fatto finora a mangiare i pomodori senza panna acida? - è il patetico quesito di mio marito. Beh, è un italiano, si capisce che la maggior parte delle metafore e dei paradossi che usa riguardano il cibo o il calcio. (Osis $2011,137,241)^{64}$

Per concludere, agli italiani che si accostano alla cultura russa può essere molto utile non solo sapere come i russi «ci vedono», ma anche da dove traggono origine i loro pregiudizi. E questo può valere, in generale, in ogni interrelazione tra popoli diversi. Conoscere gli stereotipi diffusi tra un popolo non solo aiuta a comprenderne le barzellette, ma anche a tentare di «disinnescarli» per essere visti - al di là dei propri, eventuali, tratti nazionali - semplicemente come individui.

\section{RifERIMENTI (SITOGRAFICI, FILMOGRAFICI, BIBLIOGRAFICI)}

\section{Corpus sitografico}

Ajs-nat. Turističeskoe agenstva. Ob Italii

http://www.ice-nut.ru/italy/italy249.htm

Anekdoty.ru. Anekdoty pro ital'jancev

http://anekdoty.ru/pro-italjancev/

Čao Kakao. Ital'janskij kalejdoskop. Anekdoty pro ital'jancev

http://www.ciaocacao.it/anegdot_2.htm

Italija po-russki. Forum. Anekdoty pro ital'jancev

http://italia-ru.com/forums/anekdoty-pro-italyantsev-71547

Italija. Šutlivo ob ital'jancax.

http://www.go-to-italy.ru/humor.php

Naša Italija. Portal Neapolja. Neapol' po-slavjanski. Anekdoty pro ital'jancev http://napoli1.com/forum/14-75-1

Neapol'po-slavjanski. Naša Italija, portal Neapolja. Anekdoty pro ital'jancev http://napoli1.com/forum/14-75-1

64 Merita ricordare che uno degli etnonimi peggiorativi di «italiani» è makaronniki. In Dinelli - Jampol'skaja 2008 si possono trovare conferme su questo e, in generale, su come i russi vedono gli italiani, sulla passione per Sophia Loren, Celentano, La piovra (ivi, 59-63, 109-112), ma anche curiose informazioni sugli stereotipi del maschio italiano relativamente alle sue connazionali (emancipate ed arroganti), dedite (tutte, secondo Marco Dinelli) a un «aggressivo e metodico massacro morale del giovane uomo ventiquattr'ore al giorno» (ivi, 175). 
Prima Vista. Bjuro perevodov. Počemu ital'jancy ne smejutsja nad russkimi anekdotami http://www.primavista.ru/rus/catalog/italyancy_i_anekdoty

RimIV. Anekdoty

http://rim4.ru/tema1/italjjancy-1

TurGid. Ital'jancy.. čto my o nich znaem

http://turgid.ru/countries/articles/italy/italiancy.html

Tut vsja Italija. Ital'janskij character.

http://tuttaitalia.ru/persone/carattere

Točka.net. Čem pochoži ukrajncy i tal'jancy: ТОП-5 oбščich čert

http://travel.tochka.net/7086-chem-pokhozhi-ukraintsy-i-italyantsy-top-5-obshchikhchert/

Vysokovskij.ru. Anekdoty pro ital'jancev.

http://www.vysokovskiy.ru/story/italyantsev/

\section{Filmografia}

\section{Brilljantovaja ruka}

1969, regia L.I. Gajdaj, script di L.I. Gajdaj, Ja.A. Kostukovskij, M.R. Slobodskoj, Mosfil'm, URSS.

\section{Formula ljubvi}

1984, regia di M.A. Zacharov, script di G.I. Gorin, Tvorčeskoe ob”edinenie televizionnych fil'mov, URSS.

Moskovskie kanikuly

1995, regia di A.I. Surikova, script di E.V. Braginskij, Mosfil'm, Russia.

Neverojatnye priključenija ital'jancev $v$ Rossii

1973, regia di F. Prosperi e E.A. Rjazanov, script di E.V. Bagrinskij, E.A. Rjazanov, F. Castellano, G. Mocchia, Mosfil'm - D. De Lauretis, URSS - Italia.

Priključenija kapitana Vrungelja

1976-79, regia di D.Ja. Cerkasskij, script di I.A. Vorob’ev, musica G.I. Firtič, Kievnaučfil'm, URSS.

\section{Letteratura e critica}

Allport $1976^{2}$

Bautdinov 1986

Bremmer $2005^{2}$
G.W. Allport, La natura del pregiudizio, Firenze, La Nuova Italia, $1976^{2}$ (1973) (The Nature of Prejudice, Cambridge, Mass., 1954).

G. Bautdinov, Gli italiani in Russia, Milano, Teti, 1986.

J. Bremmer, «Jokes, Jokers and Jokebooks in Ancient Greek Culture», in J. Bremmer - H. Roodenburg (eds.), A Cultural History of Humour, Oxford, Polity Press, 2005² (1997), 11- 28. 
Cortelazzo - Zolli 1992

Davies 2005

De Biasi 2006

Denisova 2012

Driessen $2005^{2}$

Gandolfo 1991

Godkewitsch 1976

Grinevski 2014

Jampol'skaja - Dinelli 2008

Lipps 2012

Mažaev 2014

Mazzara 1997

Mazzara $1998^{3}$
M. Cortelazzo - P. Zolli, «Barzelletta», in Dizionario etimologico della lingua italiana, I, Bologna, Zanichelli, 1992 (1979), 119.

Ch. Davies, «Searching for Jokes: Language, Translation, and the Cross-cultural Comparison of Humour», in T. Garfitt - E. McMorran - J. Taylor (eds.), The Anatomy of Laughter, London, Legenda, 2005, 70-85.

R. De Biasi, «I 'frames' dell'umorismo», in G. Bateson, L'umorismo nella comunicazione umana, Roma, Raffaello Cortina, 2006 (1953), 83-107.

G. Denisova, Na izlome vekov. Russkij jazyk v zerkale sovremennoj prozy, Moskva, Azbukovnik, 2012.

H. Driessen, «Homour, Laughter and the Field: Reflections from Anthropology», in J. Bremmer H. Roodenburg (eds.), A Cultural History of Humour, Oxford, Polity Press, 2005² (1997), 222-241.

G. Gandolfo (a cura di), La Russia che ride. Storielle e vignette di una vita difficile, Genova, Costa \& Nolan, 1991.

M. Godkewitsch, «Il rapporto tra il potenziale di attivazione e l'umoristicità delle barzellette», in J.H. Goldstein - P.E. McGhee (a cura di), La psicologia dello bumour. Prospettive teoriche e questioni empiriche, Milano, Franco Angeli, 1976 (1972), 187-197.

V. Grinevski, «Kapitan Vrungel' - sobiratel'nyj obraz sovetskogo proletariata», Naš fil'm.ru, http://www. nashfilm.ru/multfilms/560.html.

A. Jampol'skaja - M. Dinelli, Lenin. Dalla Pravda a Prada. Storie da una rivoluzione, Milano, TEA, 2008.

Th. Lipps, Komik und Humor. Eine psychologischästhetische Untersuchung, Marston Gate UK, CreateSpace, 2012 (1898).

A. Mažaev, «O tom, kak byl napisan fljager $\mathrm{k}$ mul'tiku o kapitane Vrungele», Chanson.ru, 15.12.14, http:// chanson.ru/about/news/istoriya_pesni_bandito_ gangsterito.

B.M. Mazzara, Stereotipi e pregiudizi, Bologna, il Mulino, 1997.

B.M. Mazzara, Appartenenza e pregiudizio. Psicologia sociale delle relazioni interetniche, Roma, Carocci, $1998^{3}$ (1996). 
Osis 2011

Pickering 2001

Pirandello 1995

Polimeni - Reiss 2006

Ramous 1984

Raskin 1985

Roberts 1994

Rylev 2015

Salmon 1998

Salmon 2003

Salmon 2004a

Salmon 2004b

Salmon 2008

Salmon Kovarski 1995
N. Osis, U samogo sinego morja. Ital'janskij dnevnik, Moskva, KoLibri, 2011.

M. Pickering, Stereotyping: The Politics of Representation, New York, Palgrave, 2001.

L. Pirandello, L’umorismo, Milano, Garzanti, 1995 (1908).

J. Polimeni - J.P. Reiss, «The First Joke: Exploring the Evolutionary Origins of Humor», Evolutionary Psychology 4 (2006), 347-366.

M. Ramous, La metrica, Milano Garzanti, 1991 (1984).

V. Raskin, Semantic Mechanisms of Humor, Dordrecht, Reidel, 1985.

E. Roberts, Russi. Se li conosci non li eviti, Torino, Sonda, 1994.

K. Rylev, «Kak sozdavalsja mul'tfil'm 'Priključenija kapitana Vrungelja'», Vesti, 27.01.2015, http://vestiukr.com/kultura/34666-kak-sozdavalsja-multfilmprikljuchenija-kapitana-vrungelja.

L. Salmon, «Nekotorye aspekty kontrastivnoj semiotiki. O ženskoj modeli v russkoj i zapadnoj kul'turach», in A.V. Pavlovskaja (gl. red.), Rossija i zapad: dialog kul'tur, V, Moskva, MGU, 1998, 452-460.

L. Salmon, «Suščestvuet li russkij jumor? K opredeleniju kategorii ‘jumorizm’ v ramkach diskussii ob universalijach v lingvokul'turach», in A. Alberti - M. Garzaniti - S. Garzonio (a cura di), Contributi italiani al XIII Congresso Internazionale degli Slavisti (Ljubljana, 15-21 agosto 2003), Pisa, AIS, 2003, 179 202.

L. Salmon, «L'antiparodia, ovvero dal 'mondo alla rovescia' all'eversione umoristica. Un approccio interdisciplinare», Moderna VI, 1 (2004), 13-29.

L. Salmon, «Il nome proprio di persona come segno di appartenenza etnica», in Ead., Quaderni di semantica XXV, 1 (2004 [2002]): L'antroponimia russa. Semiotica, pragmatica, traduzione, 39-69.

L. Salmon, Mechanizmy jumora. O tvorčestve Sergeja Dovlatova, Moskva, Progress-tradicija, 2008.

L. Salmon Kovarski, «Stereotipi ideologico-culturali. Problemi di traducibilità», in L. Pantaleoni - L. Salmon Kovarski (a cura di), Sapere linguistico e sapere enciclopedico, Bologna, CLUEB, 1995, 309-321. 
Solly 1999

M. Solli, Eti strannye ital'jancy, Moskva, Egmont Rossija, 1999 (The Xenophobe's Guide to the Italians, London, Oval Books, 1995).

Sorina 2006

M. Sorina, Voglio un marito italiano, Vicenza, Il Punto d'Incontro, 2006.

Suls 1976

J.M. Suls, «L'apprezzamento di barzellette e di cartoon in un modello a due fasi. Un'analisi del processo informazionale», in J.H. Goldstein - P.E. McGhee, La psicologia dello bumour, Milano, Franco Angeli, 1976, 110-131.

Utevskij 1996

M. Utevskij (gl. red.), Domašnjaja sinemateka. Otečestvennoe kino 1918-1996, Moskva, Dubl'-D, 1996.

van Dijk 2014

T.-A. van Dijk Ideologie. Discorso e costruzione sociale del pregiudizio, Roma, Carocci, 2014 (2003).

Villano $2013^{2}$

P. Villano, Pregiudizi e stereotipi, Roma, Carocci, $2013^{2}$ (2003).

Visani 2005

F. Visani, "An American, a French and a Russian Meet on a Desert Island...': The Representation of the Foreigner and the Cultural Transfer in Russian Contemporary Jokes», Studi Slavistici II (2005), 157 172.

Žukova 2014

O. Žukova, «Gde rodilsja neorealizm», Literaturnaja gazeta, 49/6490, 10.12.2014, http://www.lgz.ru/ article/-49-6490-10-12-2014/gde-rodilsya-neoreal$\mathrm{izm} /$. 